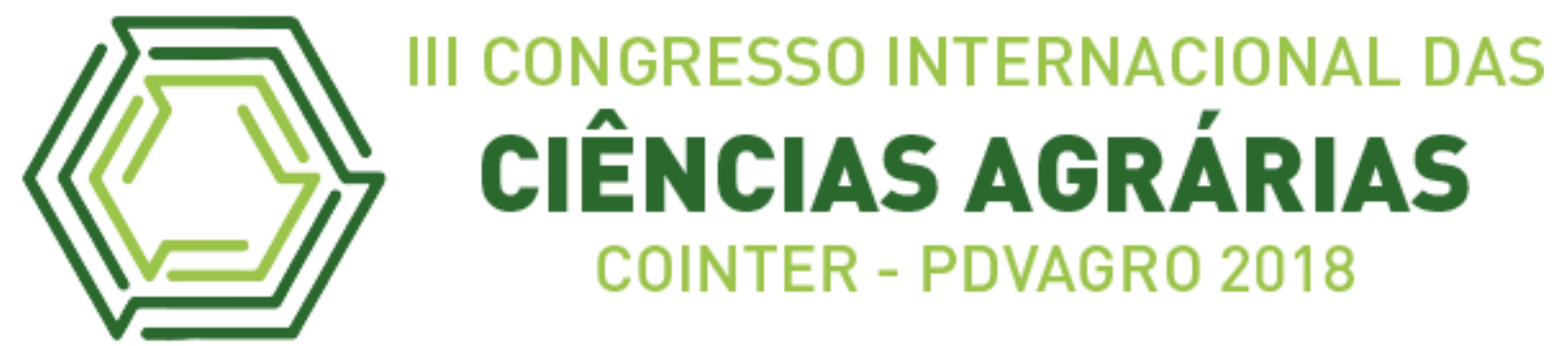

\title{
EFEITOS DE DIFERENTES DOSES DE CALCÁRIO APLICADO NO SOLO ARENOSO E ÁCIDO, PELO MÉTODO DE INCUBAÇÃO.
}

\section{EFFECTS OF DIFFERENT DOSES OF CALCARY APPLIED IN SANDY SOIL AND ACID BY THE INCUBATION METHOD.}

\author{
Apresentação: Pôster \\ Charles Eduardo Schuster ${ }^{1}$; Luis Gustavo Araujo Pereira ${ }^{2}$; \\ DOI: https://doi.org/10.31692/2526-7701.IIICOINTERPDVAGRO.2018.00454
}

\section{Introdução}

A agricultura moderna deve ser voltada ao desenvolvimento sustentável, criando e mantendo a produtividade do solo em longo prazo. Os sistemas agrícolas, de uma maneira geral, empregados no Brasil, já começaram a ser discutidos, quando relacionados aos conceitos de sustentabilidade, isto é, usar sem depredar, de modo que os recursos naturais, especialmente o solo e a água, possam ser transferidos às gerações futuras, com um legado usufruto, em condições de capacidade produtiva (NAHASS \& SEVERINO, 2003).

Grande parte dos solos brasileiros é ácida, destacando-se aqueles com vegetação de Cerrado. Esses solos são caracterizados por baixos teores de $\mathrm{Ca} 2+\mathrm{e} \mathrm{Mg} 2+$, teores elevados de $\mathrm{Al} 3+$, além da baixa disponibilidade de P. A acidez natural desses solos é proporcionada por diversos fatores, como material de origem com baixo teor de cátions básicos; precipitação pluvial maior que a evapotranspiração, acarretando lixiviação de bases no perfil (FAGERIA \& GHEYI, 1999); absorção de nutrientes catiônicos, na qual as plantas liberam quantidades equivalentes de hidrogênio $(\mathrm{H}+)$; decomposição da matéria orgânica; dissociação do gás carbônico e acidificação proporcionada pela aplicação de fertilizantes, principalmente de adubos nitrogenados.

\section{Fundamentação Teórica}

Segundo Cunha (2010), dentre os vários parâmetros químicos do solo, a determinação e correção do pH merece destaque uma vez que esta acidez, chamada de ativa, interfere na

\footnotetext{
${ }^{1}$ Bacharelado em Engenharia Agronômica, Instituto Federal do Tocantins (IFTO), E-mail: charledsch@gmail.com

${ }^{2}$ Graduado em Engenharia Agronômica, Instituto Federal do Tocantins (IFTO), E-mail: luisgustavoap@outlook.com
} 
disponibilidade dos nutrientes do solo, assim como na presença do alumínio tóxico. Solos de natureza ácida, uma vez corrigidos quimicamente apresentam grande potencial agrícola, possibilitando uma agropecuária tecnificada com elevada produtividade gerando assim mais lucros.

A prática da calagem surge como uma alternativa diante desses parâmetros, pois além de ser muito barata, traz benefícios importantíssimos à agricultura, dentre os quais se destacam a melhoria das propriedades físicas do solo, assim como as químicas e biológicas, a redução da toxidade de alguns elementos minerais, a influência na disponibilidade de nutrientes para a cultura, eliminação de elementos prejudiciais que destroem a planta, provocando assim um grande aumento na produtividade do cultivo. Apesar de tudo isso, essa prática ainda é subutilizada, tendo em vista a pouca informação recebida em nível de campo, pelos lavradores.

O solo utilizado no experimento é um solo proveniente do município de Araguatins, que se encontra numa região de transição de biomas Cerrado-Amazônico, mas apresenta características predominantes do cerrado, sendo este arenoso e com características de um solo ácido.

O presente trabalho teve por objetivo analisar os efeitos das diferentes doses de calcário aplicado no solo, observando qual seria a mais indicada para solo nessas condições, ou seja, solo arenoso e ácido, pelo método de incubação; verificar condições ideias para reação do calcário, sendo que a umidade do solo era controlada; comprovar as mudanças do pH do solo pela aplicação de corretivos.

\section{Metodologia}

O estudo com incubação de calcário foi realizado na Unidade Educativa de Produção (UEP) Agricultura 2 (AG2) do campus Araguatins do Instituto Federal do Tocantins (IFTO). O solo foi coletado na Chácara São Francisco, coordenadas, S $05^{\circ} 39^{\prime}$ 41,9', e W $048^{\circ} 46^{\prime}$ 42,8', com elevação de 122 metros, na camada de 0 a $20 \mathrm{~cm}$ de profundidade. Em seguida, o solo foi seco à sombra, destorroado, passado na peneira de 2,00 mm, logo após, separou-se 0,25 kg para a análise química e física determinando-se as frações totais de argila, areia e silte e os valores de $\mathrm{pH}\left(\mathrm{em} \mathrm{H}_{2} \mathrm{O}\right), \mathrm{M} . \mathrm{O}, \mathrm{P}, \mathrm{Na}, \mathrm{K}, \mathrm{Ca}, \mathrm{Mg}, \mathrm{Al}^{3+}, \mathrm{H}+\mathrm{Al}^{3+}, \mathrm{CTC}, \mathrm{V}$, conforme mostra as tabelas 01 e 02 abaixo. 


\begin{tabular}{|c|c|c|c|}
\hline \multicolumn{4}{|c|}{ Análise Física } \\
\hline Amostra & Areia & Argila & Silte \\
\hline \multirow{3}{*}{280} & \multicolumn{3}{|c|}{$\%$} \\
\cline { 2 - 4 } & 92,13 & 2,72 & 5,15 \\
\hline
\end{tabular}

Fonte - Laboratório de solos do IFTO.

Tabela 02 - Resultado da análise química do solo.

\begin{tabular}{|c|c|c|c|c|c|c|c|c|c|c|c|}
\hline \multicolumn{12}{|c|}{ ANÁLISE QUÍMICA DO SOLO } \\
\hline Amostra & \multirow{2}{*}{$\begin{array}{l}\mathrm{PH} \text { em } \\
\mathrm{H}_{2} \mathrm{O}\end{array}$} & \begin{tabular}{|l|l|}
$\mathbf{P}$ \\
\end{tabular} & $\mathbf{K}$ & $\mathrm{Ca}$ & Mg & Al & $\mathrm{H}+\mathrm{Al}$ & $\mathbf{S}$ & $\mathbf{T}$ & V\% & \multirow{2}{*}{$\begin{array}{l}\text { M.O. } \\
\%\end{array}$} \\
\hline № & & \multicolumn{2}{|c|}{$\mathrm{Mg} / \mathrm{dm}^{3}$} & \multicolumn{7}{|c|}{$\mathrm{cmolc} / \mathrm{dm}^{3}$} & \\
\hline 280 & 5,1 & 1,32 & 9 & 0,3 & 0,1 & 1 & 4,79 & 0,42 & 5,21 & 8,12 & 2,09 \\
\hline
\end{tabular}

Fonte - Laboratório de solos do IFTO.

O calcário utilizado tinha PRNT (Poder Relativo de Neutralização Total) de 72\% mais foi corrigido para o valor de $100 \%$, e passado pelas peneiras padronizadas pela ABNT de número 10, 20 e 50 que correspondem a 2,00, 0,84 e 0,3 mm, respectivamente.

O método utilizado para analisar as alterações de $\mathrm{pH}$ foi o de incubação de calcário, que consiste em aplicar diferentes dosagens do mesmo, cada uma em um tratamento diferente, analisando o efeito de cada dose ao fim do experimento.

O experimento foi conduzido em DIC (delineamento inteiramente casualizado), sendo um fatorial 5 x 1, constituído de cinco tratamentos com calcário com uma época de avaliação, após 30 dias a aplicação no solo, T1, T2,T3, T4 e T5 com doses de 0, 2, 4, 6 e 8 toneladas por hectare respectivamente, sendo que cada tratamento teve quatro repetições, constituindo num total de vinte unidades de experimento.

A parcela foi constituída por garrafas pets de capacidade de 2 litros, cortadas ao meio e padronizadas, com peso médio de 28 gramas cada, onde 500 gramas de solo foram homogeneizadas e colocadas para reagir com o calcário. Para determinação do percentual da capacidade máxima de retenção de água, foi colocado água nos vasos até atingir a Capacidade de Campo de $80 \%$, o que era equivalente a 564 gramas, sem contar com o peso dos vasos. O experimento foi irrigado três dias na semana por um período de um mês, logo após esse período o solo foi retirado da UEP e posto para secar a sombra, destorroado e submetido a uma nova análise de $\mathrm{pH}$.

\section{Resultados e Discussão}

O experimento foi conduzido em DIC, que é um delineamento simples, este tem como princípio a casualização e repetição. É mais utilizado em condições de laboratório, geralmente em vasos, uma vez que se for utilizado no campo, tem que certificar-se de que as condições ambientais e do material do experimento são iguais para todas as parcelas. 
De acordo com Banzatto \& Kronka (2006), a análise de variância é uma técnica que consiste na decomposição da variância total em partes atribuídas a causas conhecidas e independentes e a uma porção residual de origem desconhecida e natureza aleatória. Para essas análises, é indispensável de que se aplique o teste F, que tem por finalidade comparar estimativas de variância.

O F calculado foi significativo por ser maior que o $\mathrm{F}$ tabelado, portanto rejeita-se $\mathrm{H}_{0}$ (que é uma hipótese estatística que indica que todas as médias são iguais), ao nível de 5\% de probabilidade do teste F, como no quadro 01, abaixo. Portanto, existe pelo menos um contraste entre as médias dos resultados dos tratamentos. Enfim, as dosagens possuem efeitos diferentes nas alterações do $\mathrm{pH}$ do solo. Para saber entre quais médias existem contrastes é necessário aplicar o teste de Tukey.

\section{Quadro 01 - Análise de variância}

\begin{tabular}{|c|c|c|c|c|}
\hline CV & GL & SQ & QM & FCALC. \\
\hline TRATAMENTO & 4 & 11,0182 & 2,75455 & $38,80012 *$ \\
\hline RESÍDUO & 15 & 1,0649 & 0,070993 & \\
\hline TOTAL & 19 & 12,0831 & & \\
& & & & \\
\hline
\end{tabular}

Fonte - Elaboração própria. ( $\mathrm{F}$ tabelado $=2,90$ a $5 \%$ de probabilidade com $\mathrm{GL}$ Trat. $=4$ e $\mathrm{GL}$ do resíduo $=19$ ).

O teste de Tukey, é utilizado para testar os contrastes entre duas médias de tratamentos, estes consistem no número de combinações das médias, duas a duas. Nesse experimento, foi testado 10 contrastes de duas médias, por conter 5 tratamentos, conforme o quadro 02, abaixo. Para testar os contrastes entre as médias é necessário que elas sejam colocadas em ordem decrescente para facilitar a aplicação do teste, como no quadro 03, a seguir. Assim, pode analisar se os contrastes são significativos ou não, a 5\% de probabilidade, sendo significativa se for maior que o valor de DMS (diferença mínima significativa), que foi igual a 0,5816 .

\section{Quadro 02 - Quadro de aplicação de estimativa de contrastes entre as médias.}

$$
\begin{array}{|ll}
\mathrm{Y} 1=\mathrm{m} 5-\mathrm{m} 4=7,0125-6,6825=0,33^{\mathrm{ns}} & \mathrm{Y} 6=\mathrm{m} 4-\mathrm{m} 2=6,6825-5,835=0,8475^{*} \\
\mathrm{Y} 2=\mathrm{m} 5-\mathrm{m} 3=7,0125-6,2225=0,79^{*} & \mathrm{Y} 7=\mathrm{m} 4-\mathrm{m} 1=6,6825-4,875=1,8075^{*} \\
\mathrm{Y} 3=\mathrm{m} 5-\mathrm{m} 2=7,0125-5,835=1,1775^{*} & \mathrm{Y} 8=\mathrm{m} 3-\mathrm{m} 2=6,2225-5,835=0,3875^{*} \\
\mathrm{Y} 4=\mathrm{m} 5-\mathrm{m} 1=7,0125-4,875=2,1375^{*} & \mathrm{Y} 9=\mathrm{m} 3-\mathrm{m} 1=6,2225-4,875=1,3475^{*} \\
\mathrm{Y} 5=\mathrm{m} 4-\mathrm{m} 3=6,6825-6,2225=0,46^{\mathrm{ns}} & \mathrm{Y} 10=\mathrm{m} 2-\mathrm{m} 1=5,835-4,875=0,96^{*}
\end{array}
$$

Fonte - Elaboração própria. (ns = não significativa e * = significativo a $5 \%$ ) 
Quadro 03 - Médias em ordem decrescente

$\mathrm{M} 5=\mathrm{pH} \mathrm{7,0125}$
$\mathrm{M} 4=\mathrm{pH} \mathrm{6,6825}$
$\mathrm{M} 3=\mathrm{pH} \mathrm{6,2225}$
$\mathrm{M} 2=\mathrm{pH} \mathrm{5,835}$
$\mathrm{M} 1=\mathrm{pH} \mathrm{4,875}$

Fonte - Elaboração própria.

De acordo com o teste de Tukey, médias seguidas pela mesma letra não diferem entre si, ao nível de $5 \%$ de probabilidade. Portanto, o teste detectou uma diferença significativa entre as médias M1, M3 e M5, como mostra no quadro 04.

Quadro 04 - Representação das estimativas dos contrastes.

\begin{tabular}{|cc|c|c|c|c|c|}
\hline & & M5 & M4 & M3 & M2 & M1 \\
\hline M5 & a & - & $0,33^{\text {ns }}$ & $0,79^{*}$ & $1,1775^{*}$ & $2,1375^{*}$ \\
\hline M4 & a b & - & - & $0,46^{\text {ns }}$ & $0,8475^{*}$ & $1,8075^{*}$ \\
\hline M3 & b & - & - & - & $0,3875^{*}$ & $1,3475^{*}$ \\
\hline M2 & b c & - & - & - & - & $0,96^{*}$ \\
\hline M1 & c & - & - & - & - & - \\
\hline
\end{tabular}

Fonte - Elaboração própria.

O gráfico 01, abaixo, representa as alterações do pH em função das diferentes doses de calcário em cada tratamento, demostrando assim o efeito deste em solo arenoso, de acordo com a quantidade aplicada.

Gráfico 01 - Representação do aumento do pH em função dos tratamentos recebidos.

\section{pH final do solo em função dos tratamentos}

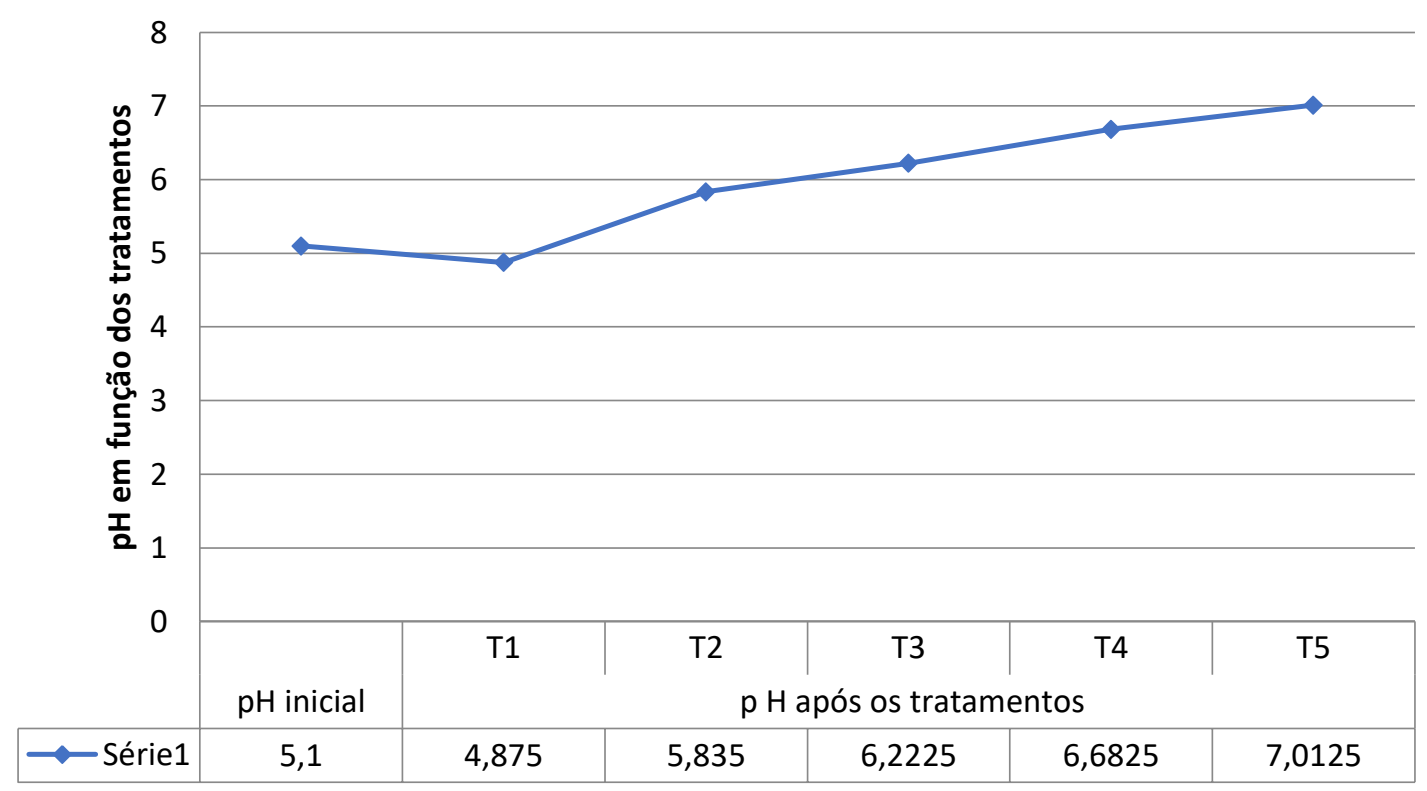

Fonte - Elaboração própria. 


\section{Conclusões}

As doses de calcário influenciaram todos os tratamentos, mas as de 2 e 4 t ha- 1 influenciaram de forma mais positiva que as demais, elevando o $\mathrm{pH}$ a uma faixa considerada ideal para o desenvolvimento de várias culturas, 5,83 e 6,22, respectivamente, além de neutralizar praticamente os teores de $\mathrm{Al}^{3+}$.

\section{Referências}

1. BANZATTO, David A. e KRONKA, Sérgio do N. Experimentação Agrícola. 4 ed. Jaboticabal: Funep,2006, 237 p.7

2. CUNHA, M. F. M. et al. Influência de diferentes métodos de recomendação de calcário no ph do solo. ENCICLOPÉDIA BIOSFERA, Centro Científico Conhecer Goiânia, vol.6, n.10, 2010 pág.5.

3. FAGERIA, N.K. \& STONE, L.F. Manejo da acidez dos solos de cerrado e de várzea do Brasil. Santo Antônio de Goiás, Embrapa Arroz e Feijão, 1999. 42p. (Embrapa Arroz e Feijão. Documentos, 92).

4. NAHASS, Samir. SEVERINO, Joaquim. Calcário Agrícola no Brasil. Série Estudos e Documentos. Rio de Janeiro: CETEM/MCT, 2003. 